\title{
Personaggio, mito, icona A League of Extraordinary Gentlemen e Penny Dreadful
}

\author{
Andrea Bellavita \\ Università degli Studi dell'Insubria, Varese \\ andrea.bellavita@uninsubria.it
}

\begin{abstract}
The League of Extraordinary Gentlemen and Penny Dreadful represent two cases of meta-finctional ucronia: the characters, belonging to a previous fictional tradition (the Victorian novel), become the protagonists of a new narrative, which follows developments and alternative settings to the original one. In the incorporation of the starting characters, which is elevated to the status of low intensity myth (Peppino Ortoleva) or cultural meme (Linda Hutcheon), and in the forms of the target text transformation, one of the possible outlines ways of generating contemporary cultural icons, through some intertextual processes: de-temporalization, re-circumstantiality, reversal of some ideological systems, hybridization and playful treatment.
\end{abstract}

\section{Keywords}

Intertextuality; Serial fiction; Adaptation; Myth; Cultural meme

\section{Sommario/Content}

1. L'icona culturale come contaminazione per adattamento stratificato

2. The League of Extraordinary Gentlemen: a parade of curiosities

3. Penny Dreadful: il potere dell'anacronismo

Bibliografia 


\section{Ocula ${ }^{22}$}

Vol 21, No 22 (April 2020) • DOI: 10.12977/ocula2020-15

Andrea Bellavita | Personaggio, mito, icona. A League of Extraordinary Gentlemen

e Penny Dreadful

\section{L'icona culturale come contaminazione per adattamento stratificato}

Riflettere sull'identità e il ruolo delle icone culturali implica la chiamata a raccolta di un dispositivo interpretativo e teorico articolato, che va dalle nozioni di immaginario ed enciclopedia, fino a quella di mito, sia nella sua dimensione storica, sia in quella desunta dalle pratiche ermeneutiche della semiotica strutturalista e post-strutturalista. In questa sede ci concentreremo su quest'ultima dimensione, proponendone una contaminazione con altre prospettive di analisi del testo mediale.

Ad arricchire un dibattito già estremamente fertile e variegato, viene in aiuto un testo recente di Peppino Ortoleva, dal titolo eloquente di Miti a bassa intensità. Racconti, media, vita quotidiana. Proseguendo sulla scorta di una tradizione che va dal Roland Barthes di Miti d'oggi all'Umberto Eco di Apocalittici e integrati, Ortoleva propone una comparazione tra una concezione classica dei miti, e una più radicata nella contemporaneità.

I primi, definiti "miti ad alta densità", sono caratterizzati da un rapporto fondato su distanza, messa in prospettiva e scostamento per differenza ed extra-ordinarietà tra l'oggetto del mito e il suo fruitore: una distanza temporale, una sovra-determinazione legata al concetto di sacro (sia come portatore di leggi e codici, che come organizzatore del rituale), e una ontologia del soprannaturale. Questo statuto di distanziamento viene superato, e anzi ribaltato, nella seconda categoria, definita "a bassa intensità": un tempo vicino a noi, storicizzato, databile e riconoscibile, un consumo libero da norme di osservanza formale, personale e de-sacralizzato, e una coincidenza (sostanziale) tra personaggi e fruitore (Ortoleva 2019: xiv-xv). Questi miti più leggeri, quotidiani, accessibili e fruibili con semplicità, trovano diritto di cittadinanza nell'ambito della circolazione mediale e della cultura pop, e potrebbero costituire una modalità stimolante per inquadrare il nostro oggetto. Senza dimenticare l'ampiezza degli studi sul fenomeno, ${ }^{1}$ proveremo a utilizzare questa intuizione come chiave interpretativa, concentrandoci su una tipologia testuale affascinante, che in qualche modo lo stesso Ortoleva sembra richiamare:

Esiste poi, sempre nell'universo delle narrazioni fantastiche, un diverso Olimpo (o Ade) contemporaneo fatto di creature della hybris o del fascino, di Peter Pan e Victor Frankenstein, di Don Giovanni o del dottor Jekyll, per citare solo alcuni nomi. Si tratta di storie, alcune di provenienza più antica altre relativamente recenti, che hanno acquisito quello che possiamo chiamare un alone mitico, a partire non, o non tanto, da un genere, quanto da un singolo testo o da un gruppo di testi. Sono narrazioni nate in origine dall'inventiva di un singolo autore ma che sono entrate in una sfera più ampia, meno soggettiva, non solo in quanto altri autori e altre forme narrative hanno continuato ad appropriarsene, e vengono continuamente ri-narrate da un medium all'altro, ma anche in quanto sono entrate nel circuito della conversazione [...]. Ci sono casi nei

1 Per una ricostruzione tassonomica dell'uso teorico del concetto di icona culturale (anche a rischio di un certo feticismo meta-critico), rimandiamo a Truman (2017). 


\section{Ocula}

Vol 21, No 22 (April 2020) • DOI: 10.12977/ocula2020-15

Andrea Bellavita | Personaggio, mito, icona. A League of Extraordinary Gentlemen

e Penny Dreadful

quali il testo originario resta ben noto come fondativo nonostante le molte vite successive, come succede, per restare ad alcuni testi otto-novecenteschi, con il Dracula di Bram Stoker, o con l’inquietante mitologia degli «Antichi» inventata dal genio bizzarro di H.P. Lovecraft, poi ripresa da altri romanzieri, e divenuta negli ultimi trent'anni oggetto di un peculiare culto online. (Ortoleva 2019: 32)

\section{E ancora:}

Nonostante la varietà (anche temporale) delle origini e dei modelli, c'è qualcosa che tiene insieme questi miti moderni? Ciascuna di queste storie fissa una figura che sembra incarnare un punto di tensione, in alcuni casi specifico del mondo moderno in altri più potenzialmente universale. [...] A differenza di quanto accade quasi sempre nei generi, dove il racconto-base è relativamente stabile ma i personaggi sono in generale mutevoli, in questo secondo tipo di miti moderni a contare sono proprio i protagonisti, non a caso dotati spesso di un nome proprio che resta nella memoria di tutti, spesso più noto e duraturo delle stesse storie che indossa. (Ortoleva 2019: 33)

Emergono tre caratteri essenziali: l'origine letteraria, l'apertura ad una ri-narrazione costante, che valicando il medium di partenza consente l'allargamento a registri più popolari (chiaramente: dal libro al film, alla televisione, al fumetto, alla video-ludica...), fino alla semplice condivisione interpersonale, e la centralità del personaggio. Ci troveremmo dunque di fronte a personaggi letterari dal potenziale mitico così forte da sviluppare una vita mediale totalmente autonoma dalla loro esistenza letteraria, suscettibili di cambiare pelle nel passaggio tra un'incarnazione testuale e un'altra. Una tipologia assimilabile a quella che Linda Hutcheon, assorbendo la terminologia del genetista Richard Dawkins (1976), definisce come memi, strutture narrative in grado di muoversi diacronicamente e transmedialmente, in virtù della propria efficacia strutturale e grazie a una costante ri-scrittura che ne rafforzano progressivamente la tenuta e la capacità di essere assorbiti e metabolizzati da diversi sistemi socioculturali. ${ }^{2}$

Non casualmente i riferimenti espliciti a storie, e personaggi, letterari hanno evidenti tratti in comune con la proposta di Ortoleva:

Come accade per gli esseri viventi, a sopravvivere sono le storie in grado di adattarsi meglio delle altre (attraverso la mutazione) a un dato ambiente: quelle di Carmen,

2 «Per quanto Dawkins scrivendo dei memi pensasse in realtà alle idee, anche le storie in un certo senso sono idee, e si può dire che funzionino nello stesso modo. Alcuni di esse si rafforzano attraverso la sopravvivenza (persistenza in una data cultura) o la riproduzione (numero di adattamenti). Gli adattamenti, come l'evoluzione, sono un fenomeno transgenerazionale. Alcune storie hanno ovviamente, per dirla con Dawkins, maggiore "stabilità e capacità di penetrazione nell'ambiente culturale". Le storie riescono ad essere raccontate e riraccontate in modi diversi, in nuovi contesti culturali e in diverse materie dell'espressione; come i geni, si adattano ai nuovi ambienti in virtù della loro capacità di mutazione - nei loro "discendenti" e cioè nei loro adattamenti. E le storie più forti e poste nelle migliori condizioni fanno ben più che sopravvivere, fioriscono» (Hutcheon 2006: 60). 


\section{Ocula}

Vol 21, No 22 (April 2020) • DOI: 10.12977/ocula2020-15

Andrea Bellavita | Personaggio, mito, icona. A League of Extraordinary Gentlemen

e Penny Dreadful

Don Giovanni, Don Quijote, Robinson Crusoe, Dracula, Amleto e così via. Con le parole di Richard Dawkins, «alcuni memi hanno più successo di altri nel pool memico». (Hutcheon $2006: 236$ )

Nell'alveo di questi paradigmi interpretativi, avanziamo dunque l'ipotesi che una delle modalità di creazione di un'icona culturale, o più precisamente di un'icona pop, sia la stratificazione di adattamenti progressivi a partire da un personaggio letterario originale (un mito a bassa intensità, o un meme), che allontanandosi, anche in maniera critica, dall'identità di base, consenta una costante contaminazione con il frame temporale e culturale che lo ospita.

Per illustrare questo processo di contaminazione per adattamento stratificato, prenderemo in esame due testi esemplari che costituiscono, per così dire, dei termini ad quem esplicitamente meta-testuali del processo: la serie di comic book The League of Extraordinary Gentlemen e la serie televisiva Penny Dreadful.

\section{The League of Extraordinary Gentlemen: a parade of curiosities}

The League of Extraordinary Gentlemen, scritto da Alan Moore e disegnato da Kevin O'Neill, è un oggetto di non facile collocazione, che si estende, attraverso una serie di pubblicazioni autonome, dal 1999 al 2019. Il codice rappresentativo principale è quello del fumetto, per quanto le inserzioni sempre più rilevanti di brani scritti (sempre in qualche modo parodici o meta-narrativi rispetto a forme e tradizioni consolidate) e altre forme iconografiche (locandine, cartoline illustrate, pubblicità, riproduzioni di annunci, periodici, giochi da tavola...), imponga di considerarlo come un progetto omogeneo di worldbuilding pluri-testuale. Non, curiosamente e in completa contro-tendenza con l'evoluzione dell'ecosistema contemporaneo, cross-mediale o trans-mediale: l'unica forma di adattamento al di fuori della pagina scritta è rappresentato dal film omonimo (The League of Extraordinary Gentlemen, S. Norrington, 2003), autentico tradimento dell'originale piuttosto che traduzione, ampiamente disconosciuto da Moore. Vale la pena, dunque, di ripercorrerne nei suoi tratti essenziali l'evoluzione editoriale.

La prima apparizione è composta da sei uscite, pubblicate tra il marzo del 1999 e il settembre 2000 dall'etichetta America's Best Comics negli USA e dall'etichetta Vertigo in UK. ${ }^{3}$ È importante, anche ai fini della nostra analisi,

3 Un'ulteriore precisazione è d'obbligo: America's Best Comics nasce come etichetta pensata appositamente per consentire ad Alan Moore di sviluppare progetti autonomi e fuori dagli schemi classici del fumetto mainstream. Viene creata all'interno di Wildstorm, progetto fondato da Jim Lee nel 1991 come studios interno dell'Image Comics, a sua volta casa editrice indipendente creata da autori fuoriusciti dai due principali marchi statunitensi, Marvel e DC Comics, per gestire personaggi autoprodotti e fuori dai diritti degli universi predominanti. Proprio mentre Moore sta sviluppando alcuni delle sue linee narrative, Wildstorm, e di conseguenza ABC, vengono vendute alla DC Comics. Moore decide comunque di proseguire, almeno per qualche anno, nella sua collaborazione con DC. Il pubblico italiano ha potuto conoscere le prime sei uscite 


\section{Ocula ${ }^{22}$}

Vol 21, No 22 (April 2020) • DOI: 10.12977/ocula2020-15

Andrea Bellavita | Personaggio, mito, icona. A League of Extraordinary Gentlemen

e Penny Dreadful

sottolineare come la prima forma di "somministrazione" al pubblico sia stata episodica e seriale, con inviti diretti (e ludici) a continuare la lettura e contenuti extra (cover alternative, un gioco da tavolo, card da collezionare acquistando pacchetti di sigarette da un penny). La pubblicazione in volume di The League of Extraordinary Gentlemen Volume I è dunque successiva. Analoga è la forma della seconda parte del racconto, in sei uscite tra il settembre 2002 e il novembre 2003, raccolte come The League of Extraordinary Gentlemen Volume II. Il terzo volume, The League of Extraordinary Gentlemen: Black Dossier, esce come corpo autonomo nel novembre del 2007, ancora per la DC Comics. Successivamente vengono pubblicati tre distinti volumetti di 72 pagine, questa volta per la Top Shelf Production: Century 1910 (2009), Century 1969 (2011) e Century 2009 (2012), poi raccolti nel volume collettaneo The League of Extraordinary Gentlemen, Volume III: Century. Ad esso seguiranno, con la stessa modalità, tre spin-off: Nemo: Heart of Ice (2013), Nemo: The Roses of Berlin (2014), Nemo: River of Ghosts (2015). L'ultimo arco narrativo, appena pubblicato, segue la modalità dei primi due: The League of Extraordinary Gentlemen, Volume IV: Tempest.

In estrema sintesi cercheremo di rendere ragione dell'intricata rete di personaggi e di relazioni contenute nei primi due archi narrativi, che sono anche i più omogenei, perché prevedono una sostanziale unità di luogo e una continuità temporale che si risolve in poche settimane. Nel primo volume, ambientato in una Londra immaginaria del 1898, un agente del servizio segreto britannico, l'M15, che risponde al nome di Mr. Campion Bond (e che si intuisce essere il nonno del più celebre James Bond), convoca Mina Murray (dal Dracula di B. Stoker, 1897) con il compito di arruolare un gruppo di personaggi stravaganti (la lega del titolo) per salvare l'Impero dai pericoli che incombono. ${ }^{4} \mathrm{La}$ missione consiste nello smantellare il diabolico piano ordito dal Dottore (un cinese a capo dell'intera criminalità dell'East End, ispirato al Fu Manchu dei romanzi di Sax Rohmer), intenzionato a conquistare il mondo con macchine volanti alimentante dalla cavorite, una sostanza di provenienza aliena. Su indicazione di Mr. Bond, Mina Murray, deve dunque arruolare Allan Quatermain (esploratore e cacciatore dei romanzi di Henry Rider Haggard, a partire da Le miniere di re Salomone, 1885), il Capitano Nemo (scienziato, esploratore e pirata anti-britannico, con il suo sottomarino Nautilus, creato da Jules Verne in Ventimila leghe sotto i mari, 1874), Henry Jekyll (dal romanzo di Louis Stevenson, 1886, catturato a Parigi nella forma della sua nemesi Mr. Hyde, grazie all'aiuto di Auguste Dupin...) e Hawley Griffin (che nel romanzo L'uomo invisibile di H.G Wells, 1897, si chiama Jack5). A capo dell'M15 è il misterioso M, che si rivelerà essere il Professor Moriarty (la nemesi di Sherlock

di TLoEG attraverso una pubblicazione omonima (America's Best Comics) che raccoglieva periodicamente i nuovi capitoli di diverse linee narrative di Moore: Promethea, Tom Strong...

4 È lo stesso Mr. Bond a definire il gruppo come parade of curiosities (serraglio nella versione italiana), durante il suo primo incontro con Mina Murray.

5 Il cambiamento di nome dovrebbe rimandare a Hawley Harvey Crippen, medico e assassino statunitense, condannato a morte nel 1910 per l'omicidio della moglie. 


\section{Ocula}

Vol 21, No 22 (April 2020) • DOI: 10.12977/ocula2020-15

Andrea Bellavita | Personaggio, mito, icona. A League of Extraordinary Gentlemen

e Penny Dreadful

Holmes, comparso nel racconto L'ultima avventura di Arthur Conan Doyle, 1893), sopravvissuto alla caduta alle cascate di Reichenbach e intenzionato a impadronirsi della cavorite. Per i primi cinque episodi i componenti della lega sono convinti di lavorare per Mycroft Holmes (il fratello di Holmes), che prenderà effettivamente il posto di Moriarty, dopo la sua morte.

In quest'ultimo episodio, il gruppo si trova a fronteggiare un'invasione di tripodi marziani (da La guerra dei mondi, H.G. Wells, 1897), e riesce nell'impresa grazie a un'arma batteriologica fornita dal dottor Moreau (L'isola del dottor Moreau, H.G. Wells, 1896). Alla fine dell'arco narrativo, il gruppo di scioglie: Griffin viene ucciso da Hyde dopo aver tradito i compagni ed essersi alleato con i marziani, lo stesso Hyde si sacrificherà in un ultimo scontro contro i tripodi per consentire agli altri componenti di sferrare l'attacco finale, Nemo lascerà Londra disgustato per l'uso di un'arma di distruzione di massa, e Mina Murray si ritirerà in solitudine, dopo aver lasciato Quatermain (con il quale aveva iniziato una relazione), seduto su una panchina nel parco. Il tutto in stile steampunk.

La semplice enunciazione delle linee narrative non rende minimamente ragione della mirabolante creatività e complessità (di rimandi enciclopedici, riferimenti, particolari, dettagli) dispiegate da Moore e O'Neill. ${ }^{6}$ Almeno un punto però appare da subito chiarissimo: Moore raccoglie nella sua opera gran parte dei protagonisti, e degli immaginari, del romanzo vittoriano di fine Ottocento. Non soltanto riconosce ai suoi protagonisti il valore mitico cui fa riferimento Ortoleva, e la funzione di meme che Hutcheon stabilisce alla base di ogni possibile adattamento, ma ne rafforza il potenziale attraverso l'ipotesi di una convivenza testuale, sottoponendoli a un doppio registro di riscrittura, che arriva a fornire loro una identità completamente nuova.

La prima è connaturata al macro-genere della crossover fiction, che comprende forme testuali molto differenti tra loro (per tipologia, registro, medium) caratterizzati dalla compresenza e dall'incrocio di universi narrativi originali, e si definisce come un ipertesto generato da una pluralità (almeno due) di ipotesti autonomi. ${ }^{7}$ In questo caso le matrici letterarie sono moltissime, e si arricchiranno progressivamente nei capitoli successivi dell'opera. ${ }^{8}$

Se la crossover fiction è una tendenza sempre più diffusa all'interno dell'ecosistema mediale contemporaneo (e forse uno dei tratti definitori della postmodernità), la dimensione gruppale rimanda a una fattispecie molto più ridotta, ma ancora più affascinante, che altrove ho definito ucronia meta-finzionale (Bellavita 2019: 248), e che descrive uno sviluppo alternativo ai singoli apparati diegetici degli ipotesti, fondato sulla convergenza (l'incontro) dei personaggi principali, che deviano dal loro percorso narrativo originale (sem-

6 Per un interessante, e divertente, viaggio iniziatico in questo parco dei divertimenti letterario, si rimanda a Nevins (2003, 2004).

7 Per un'interpretazione più strettamente narratologica del fenomeno, mi permetto di rimandare a Bellavita (2019).

8 Nei diversi volumi verranno presentate, descritte, o semplicemente evocate, diverse altre league, impegnate in analoghe missioni in tutte le epoche storiche. 


\section{Ocula}

Vol 21, No 22 (April 2020) • DOI: 10.12977/ocula2020-15

Andrea Bellavita | Personaggio, mito, icona. A League of Extraordinary Gentlemen

e Penny Dreadful

pre accettato come parallelo, o almeno precedente all'intreccio in questione) per portare a termine un nuovo progetto narrativo di gruppo.

Il modello di riferimento è sicuramente quello della Wold Newton family, alla base di tre romanzi dello scrittore di fantascienza americano Philip José Farmer: Tarzan Alive: A Definitive Biography of Lord Greystoke (1972), Doc Savage: His Apocalyptic Life (1973) e The Other Log of Phileas Fogg (1973). Il concept fondativo del ciclo prevede che la caduta di un meteorite (il Wold Cottage meteorite, realmente rinvenuto nello Yorkshire nel 1795) abbia provocato una mutazione genetica nei discendenti degli abitanti del villaggio, rendendoli "straordinari" e dotati di capacità e competenze superiori alla media. ${ }^{9}$ In Farmer emerge già l'idea che personaggi che condividono l'appartenenza a diverse forme del racconto popolare possano sperimentare una convivenza autonoma in una nuova struttura letteraria, ma non c'è alcun intento trasformativo, valoriale o culturale, al di fuori del puro entertainment. Un passo ulteriore, sulla linea della ridefinizione identitaria, è rappresentato dal meta-romanzo giallo-umoristico La verità sul caso D. di Fruttero \& Lucentini (1989), in cui si immagina un convegno internazionale incaricato di completare le più famose opere incompiute della musica e della letteratura: nella sezione dedicata a Il mistero di Edwin Drood di Charles Dickens, si ritrovano Sherlock Holmes e il Dr. Watson, Padre Brown, il Commissario Maigret, Auguste Dupin, Philip Marlowe e Lew Archer, Nero Wolfe, Hercule Poirot e Porfirij Petrovič. In questo caso è evidente l'intento ironico (e dissacratorio), che nasce dalle collisioni (di metodo, di stile, di modus operandi) tra i diversi investigatori, costretti loro malgrado a confrontarsi e condividere, seppur parzialmente, uno spazio e un tempo.

La rimodulazione dei tratti identitari è invece essenziale nella scrittura di Moore,${ }^{10}$ dove ciascun personaggio subisce un totale capovolgimento valoria-

9 Oltre ai tre protagonisti dei romanzi, la WN family è arrivata a comprendere molti altri personaggi di finzione, più o meno noti, di volta in volta implicati in relazioni dirette: Sherlock Holmes e il Professor Moriarty (fino al meno fortunato eroe di Conan Doyle, il Professor Challenger), Allan Quatermain, Fu Manchu e Sir Denis Nayland Smith, Richard Hannay (reso celebre dall'adattamento cinematografico The 39 Steps di Alfred Hitchcock), i ladri gentiluomini A.J. Raffles e Arsène Lupin, il viaggiatore de La macchina del tempo di H.G. Wells, detective e investigatori privati (dall'antesignano Monsieur Lecoq a Sam Spade e Philip Marlowe, Nero Wolf, Bulldog Drummond, Lew Archer, Travis McGee), agenti segreti (Mr. Moto e James Bond), protagonisti di pulp fiction degli anni Trenta (G-8, The Shadow, The Spider, The Avenger), fino a una serie di characters più laterali (il detective Lord Peter Wimsey, lo spadaccino Salomon Kane, il medico avventuriero Captain Blood, la Primula Rossa, e il suo omologo Gray Seal).

10 Tutta l'opera di Moore è caratterizzata da un lavoro costante di rilettura e reinterpretazione di immaginari narrativi o radicati nell'immaginario collettivo: $V$ for Vendetta (il riferimento all'icona di Guy Fawkes), Capitan Bretagna e Miracleman (ripensamenti britannici e decostruzionisti degli americani Capitan America e Capitan Marvel), Watchmen (messa in crisi di tutto l'universo supereroistico) e Tom Strong (ibrido tra Tarzan e Doc Savage e omaggio-rilettura dei personaggi dei pulp magazine, analogamente a Tomorrow Stories), Supreme (rilettura in chiave moderna di Superman). Ancora più esplicitamente From Hell (sui delitti di Jack lo Squartatore), Lost 


\section{Ocula ${ }^{22}$}

Vol 21, No 22 (April 2020) • DOI: 10.12977/ocula2020-15

Andrea Bellavita | Personaggio, mito, icona. A League of Extraordinary Gentlemen

e Penny Dreadful

le: Mina Murray diventa una leader carismatica, Allan Quatermain un vecchio pavido e oppiomane, Griffin uno psicopatico, Nemo un assassino sanguinario e Hyde dimostra di poter controllare i propri istinti, e di essere capace anche di sensibilità ed eroismo. Il lavoro di adattamento dei personaggi originali assume i tratti di una ridefinizione profonda, che guarda contemporaneamente al passato (il modo in cui quelle forme mitiche restituivano una messa in forma valoriale della società) e al presente (il modo in cui ci rapportiamo con un'ideologia storicizzata attraverso una codifica narrativa), e al naturale cortocircuito tra le due dimensioni temporali (come possono trovare spazio, oggi, archetipi narrativi così indiziari?). Nel riscrivere l'azione dei suoi personaggi nel 1898, Moore sembra chiedersi piuttosto: come si comporterebbero oggi quegli stessi uomini e donne? Spiega bene a tal proposito Alison Halsall:

In their appropriation and re-envisioning of characters from Victorian literature and culture, Moore and O'Neill focus on received notions of the period - sexuality, heroism, identity, and Empire - to delight in the social and political contradictions inherent in these fantasies, all seen through their present-day lens [...]. Presenting the cusp of a new century serving beautifully to highlight the ideological sea change occurring, these two volumes parody popular conceptions of the socially conservative Victorian period and Victorian literature by fixing on issues like femininity, Empire, and the construction of monstrousness that have been, and, to a certain extent, still are being interrogated in literary criticism. (Halsall 2015: 253-254)

Come molti altri esponenti dei new-victorian studies, ${ }^{11}$ Halsall si concentra in particolare sulla funzione narrativa di Mina Murray, autentica social monster (ha perso la verginità con il vampiro Dracula, è divorziata, e quindi esclusa dalla società), che è insieme iperbole metaforica della marginalizzazione del femminile in età vittoriana, e superamento secondo i codici socio-culturali contemporanei. L'unico personaggio letterariamente "cadetto" (in fondo, a differenza dei suoi compagni di avventura, non è la protagonista eponima del romanzo che l'ha generata), diventa centro propulsore delle attività della lega, unica donna in grado di mantenere un atteggiamento razionale e risolutivo, e anche unica a saper gestire consapevolmente la propria sessualità, attorniata da un erotomane pervertito (Griffin) e uomini frustrati incapaci di affrontare la propria doppiezza (l'omosessualità latente di Jeckyll) o la perdita della virilità (Quatermain). Unica, infine, capace di mitigare la violenza predatoria di Hyde, costringendolo ad un gesto di tenerezza (la richiesta di poterle sfiorare un seno, prima della missione suicida con i droni). La (ri)costruzione di Mina come icona culturale, passa dunque attraverso una serie di attività trasformative: prima il riconoscimento del suo ruolo originale (nel Dracula di Stoker)

Girls (il ricordo delle avventure erotiche di Alice di Le avventure di Alice nel Paese delle Meraviglie, Dorothy da Il meraviglioso mago di Oz e Wendy da Peter Pan), Promethea (rilettura in chiave occulta della mitologia greca), The Courtyard, Neonomicom e Providence (a partire dall'universo di H.P. Lovecraft), e Fashion Beast (adattamento di La bella e la bestia).

11 Si veda a questo proposito almeno: Jones (2010), Wegner (2010), Domsch (2012). 


\section{Ocula}

Vol 21, No 22 (April 2020) • DOI: 10.12977/ocula2020-15

Andrea Bellavita | Personaggio, mito, icona. A League of Extraordinary Gentlemen

e Penny Dreadful

come rappresentazione indiziaria di un modello di femminilità ottocentesca, poi l'esplicitazione dei tratti lasciati sotto-traccia (in chiave metonimica: il rapporto sessuale con Dracula) e quindi la re-iscrizione in un nuovo racconto come eroina postmoderna.

Il riferimento all'immaginario vittoriano è però uno solo dei poli dell'intricata intelaiatura di cooptazione testuale che Moore predispone intorno ai suoi personaggi. Si diceva in precedenza dell'importanza della pubblicazione seriale del primo arco narrativo e della disseminazione di altre forme di comunicazione appartenenti all'industria culturale: è emblematica in questo senso la tavola extra pubblicata sul secondo numero, in cui i volti dei personaggi sono rappresentati sotto forma di card da collezione, ma recano ciascuno, come "data di nascita", l'anno della propria pubblicazione editoriale. ${ }^{12}$ La loro identità romanzesca viene immediatamente contaminata da una modalità di rappresentazione non soltanto popolare ma, potremmo dire, sub-culturale ed extra-letteraria. Ciascuno dei numeri si conclude con un richiamo diretto al "giovane lettore" a pazientare per il tempo necessario alla prossima uscita, prospettandogli mirabolanti ed eccitanti sviluppi. ${ }^{13}$ Il registro, e l'esplicitazione (totalmente ludica: il target di riferimento dell'opera è senza dubbio più adulta) dell'identità del lettore esplicita l'altro grande universo di riferimento di Moore: la letteratura d'appendice, popolare e sensazionalistica, dei penny dreadful. ${ }^{14}$

Lungo questi due binari, di tradizione letteraria e cultura popolare (che costituiscono da sempre l'orizzonte del suo lavoro), Moore riesce a eternaliz$z{ }^{15}{ }^{15}$ i suoi personaggi, a renderli vettori di comprensione di diverse epoche e di diversi sistemi sociali. A renderli icone culturali trans-temporali.

12 Per un approfondimento, e una contestualizzazione storico-critica, si veda Mitchell (2017: 258).

13 A titolo esemplificativo: «Nel prossimo episodio del nostro periodico illustrato, troverete ulteriori scene per il vostro svago e la vostra sorpresa, incluso un episodio di natura oscena che le nostre Lettrici, possedendo una più delicata sensibilità, potrebbero voler evitare»; «Nella prossima storia del nostro Romanzo illustrato per ragazzi proseguiremo con questo avvincente racconto, in cui i migliori agenti dell'impero si troveranno in conflitto con lo scaltro Cinese, accompagnati da un'ampia varietà di illustrazioni a colori del nostro artista, che risulteranno certamente emozionanti per i virili e smaliziati giovani d'oggi».

14 Sul legame con l'immaginario dei penny dreadful si veda Toss (2015), Sulmicki (2011); per un inquadramento storico del fenomeno dei penny dreadful, e soprattutto per una focalizzazione sul ruolo del pubblico giovane per lo sviluppo dell'editoria ottocentesca, si veda Springhall (1994).

15 Il riferimento diegetico alla loro immortalità diventerà sempre più esplicito: all'inizio di Black Dossier ritroviamo Mina Murray e Allan Quatermain nel 1958, perfettamente intatti dopo essersi bagnati nella fonte dell'eterna giovinezza in Congo, e un peso sempre più rilevante sarà ricoperto da Orlando, il transgender eterno di Virginia Woolf. Di tutti i componenti delle diverse leghe, i tre rimarranno gli unici superstiti, protagonisti dei tre capitoli compresi in Century, dal 1910 al 2009: al centro del secondo, ambientato nel 1969, sarà poi il tema della trasmigrazione delle anime e delle identità, che consente a personaggi del passato (è il caso dell'occultista Oliver Haddo, protagonista del romanzo The Magician, di William Somerset Mugham, e ispirato alla figura di Aleister Crowley) di possedere, letteralmente, i corpi finzionali di nuovi characters. 


\section{Ocula}

Vol 21, No 22 (April 2020) • DOI: 10.12977/ocula2020-15

Andrea Bellavita | Personaggio, mito, icona. A League of Extraordinary Gentlemen

e Penny Dreadful

\section{Penny Dreadful: il potere dell'anacronismo}

La serie televisiva ${ }^{16}$ Penny Dreadful, coprodotta dalla premium cable tv statunitense Showtime e dalla britannica Sky Atlantic per tre stagioni e un totale di 27 episodi, dal 2014 al 2016, rappresenta senza dubbio un testo derivato (o almeno ispirato) da The League of Extraordinary Gentlemen, per quanto il creatore John Logan non abbia mai voluto considerarla esplicitamente come un adattamento, rimandando piuttosto all'origine comune dei romanzi di Farmer. ${ }^{17}$ È sufficiente però una breve presentazione dell'ambientazione e dei personaggi principali per individuarne i tratti comuni, sempre compresi tra i due immaginari fondativi: il romanzo vittoriano e la letteratura popolare britannica (fin dal titolo). Nella Londra del 1891, Sir Malcolm Murray (esploratore in Africa, tratteggiato sui caratteri di Allan Quatermain), cerca di liberare la figlia Mina, rapita dai vampiri, con l'aiuto di Miss Vanessa Ives (la migliore amica di Mina), di Ethan Chandler (pistolero americano in viaggio per l'Europa con un circo viaggiante, ispirato a Buffalo Bill, e segretamente licantropo) e del giovane Dott. Victor Frankenstein. Intorno a loro: Abraham Van Helsing (che ha il compito di introdurre il giovane Victor ai segreti del vampirismo), Dorian Gray, la Creatura di Frankenstein, e la prostituta tubercolotica Brona Croft, che rinascerà come Lily Frankenstein, Henry Jekyll e Mr. Hyde, il Reinfield di Dracula.

Se il lavoro di riscrittura degli archetipi vittoriani è sostanzialmente analogo, ${ }^{18}$ l'appropriazione dei miti a bassa densità operato da Logan si muove però a un livello ulteriore rispetto a quello di Moore, perché non si limita a ridefinire l'identità degli originali letterari, ma opera sempre uno spostamento meta-testuale. Il caso più interessante è quello di Vanessa Ives, che anche in questo caso rappresenta il fulcro simbolico e diegetico dell'intera narrazione: Vanessa non è Mina Murray ma viene presentata come la sua migliore amica, una sorta di nuova versione della Lucy Westenra del romanzo di Stoker, di cui però si rivela essere un ribaltamento speculare. ${ }^{19} \mathrm{Al}$ contrario di Lucy, che viene "sacrificata" diegeticamente in Dracula, per consentire la sopravvivenza di Mina, Vanessa provoca la perdizione dell'amica, concupendone il promesso sposo la vigilia delle nozze, e conducendola prima alla disperazione e poi

16 Per una, minima, contestualizzazione dell'evoluzione della fiction contemporanea, si rimanda a Mittel (2015), Pescatore (2018).

17 La carriera di sceneggiatore di John Logan è costellata di incursioni nella ri-scrittura di mitologie e mitografie: dalla storia (Il gladiatore, 2000; L'ultimo samurai, 2003; The Aviator, 2004) alla cultura pop (il suo Sweeney Todd, 2007, è un esplicito omaggio a un topos del penny dreadful), dalla saga cinematografica (Star Trek, oo7 e Alien) fino al puro meta-cinema (Hugo Cabret, 2011).

18 Si veda a questo proposito: Baptista do Lago (2015), Gagliardi (2016), Artt (2018), García Sahagún e Arquero Blanco (2019).

19 «[...] both works present two seemingly opposite women: Lucy Westenra and Mina Murray in Dracula and Vanessa Ives and Mina Murray in Penny Dreadful. [...] Through its adaptation of Dracula, Penny Dreadful illustrates Victorian cultural anxieties regarding women's selfhood and identities in relation to societal stability» (Rocha 2016: 30-31). 


\section{Ocula}

Vol 21, No 22 (April 2020) • DOI: 10.12977/ocula2020-15

Andrea Bellavita | Personaggio, mito, icona. A League of Extraordinary Gentlemen

e Penny Dreadful

alla possessione (il rapimento) vampiresco, in una sorta di vendetta postuma, che affranca completamente il topos del femminile vittoriano, restituendole il potere che deriva (ancora, come nella Mina di Moore) dalla sicurezza con cui dispone della propria sessualità.

Nel quarto episodio della prima stagione Vanessa incontra (idealmente) la propria identità letteraria sotto le sembianze della piccola orfana Lucy, che la interpella sul significato della vita e della morte. I riferimenti tra la pura e dolce Lucy Westenra di Stoker e la sensuale e perversa Vanessa di Penny Dreadful sono continui, ma sempre deviati, in qualche modo contaminati e perturbati: Lucy era contesa da tre pretendenti (Arthur, figlio di Lord Godalming, Quincey Morris, un cowboy americano, e il dottor John Seward, precursore della psichiatria), mentre Vanessa è stata promessa sposa di Peter, figlio di Sir Malcolm, e vive ambigue relazioni con il cowboy Ethan e con il giovane dottor Frankenstein (mentre Seward troverà un suo corrispettivo femminile nella terza stagione).

Alison Lee e Frederick D. King, in quella che è la più attenta e acuminata lettura critica della serie, ricorrono esplicitamente ai concetti di mito e di meme culturale: ${ }^{20}$

Penny Dreadful's practice of adaptation disrupts linearity and undermines notions of the authority and priority of an originating text. The original is contaminated by a history of adaptations that have transformed Mary Shelley's Frankenstein (1818, 1831), Oscar Wilde's The Picture of Dorian Gray (1890, 1891), and Bram Stoker's Dracula (1897) into vehicles of cultural transmission: memes that have come to redefine history as a myth of modernity. [...] In effect, Penny Dreadful is a form of non-linear cross-contamination that destroys the metanarrative of the originary text. Contamination also becomes a means of breaking down the barrier between the past and present, transforming classic texts into contemporary cultural iconography. [...] Penny Dreadful is not so much an adaptation of the classic novels, as it is an adaptation of what those novels would look like had they been plagiarized into penny dreadfuls, and then read through subsequent and substantial cultural referents like Buffy, Twilight or 1950s Hammer studio films. The genetic line, then, is contaminated and confused. [...] The series does not treat them as classic British novels so much as treat them as cultural memes that continue to live in contemporary culture as much as they did in nineteenth-century British literature. In other words, the past contaminates the present. (Lee e King 2015)

$\mathrm{Al}$ di là dell'infinita serie di riferimenti all'enciclopedia letteraria (e mediale, cinematografica in particolare) pertinente, sempre sospesi tra sberleffo postmoderno ${ }^{21}$ ed esibizione colta per un pubblico di nicchia, c’è un elemento

20 Sull'impiego del concetto di meme culturale, si veda anche Braid (2017).

21 Uno su tutti: nel sesto episodio della prima stagione, Van Helsing mostra a Victor Frankenstein una pubblicazione di Varney the Vampire, un penny dreadful pubblicato dal 1845 al 1847, che definisce fantasiosa ma utile come "vulgata del folklore balcanico" per comprendere il fenomeno del vampirismo. Van Helsing compare letterariamente soltanto cinquant'anni dopo, nel 1897, e dunque "non potrebbe" conoscere, 


\section{Ocula}

Vol 21, No 22 (April 2020) • DOI: 10.12977/ocula2020-15

Andrea Bellavita | Personaggio, mito, icona. A League of Extraordinary Gentlemen

e Penny Dreadful

che differenzia profondamente Penny Dreadful da The League of Extraordinary Gentlemen: il ricorso costante e compiaciuto all'aggiornamento anacronistico. Se Moore si affidava esclusivamente all'ambientazione steampunk per descrivere la sua ucronia vittoriana, Logan ammicca all'immaginario contemporaneo dello spettatore.

Le occorrenze più evidenti ruotano ancora intorno al personaggio di Dorian Gray, che con Vanessa intreccia una torbida relazione sessuale. L'icona del dandy vittoriano ha comportamenti e abbigliamento totalmente fuori dal tempo: veste camicie aperte a collo rialzato, pantaloni di pelle, collane di pietre preziose e metallo, ma non indossa mai il cappello e i guanti (durante la seduta spiritica del secondo episodio, alla richiesta di togliersi i guanti, per gli uomini, e i gioielli, per le donne, Dorian e Vanessa sono gli unici ad essere già pronti, perché fuori dagli schemi), l'orgia che organizza nella sua dimora nel quarto episodio ha l'estetica della pubblicità di un profumo, tra musica, cori che si intrecciano su divani di broccato, grandi immagini che occhieggiano dalle pareti. Nello stesso episodio le due coppie di amanti (Ethan e la prostituta Brona e Dorian e Vanessa) si incontrano a una rappresentazione del Grand Guignol (che non ha mai avuto, storicamente, una sede londinese): se lo scambio di sguardi dai palchi dei due borghesi britannici rimanda a $L e$ relazioni pericolose di Stephen Frears, lo stupore ingenuo della prostituta invitata a teatro dal ricco rampollo statunitense (Ethan, che è letteralmente un lupo mannaro americano a Londra, fugge da un padre troppo ricco, e troppo esigente) è una citazione divertente e divertita di Pretty Woman.

Dorian è affascinato dalla tecnologia, quella del grammofono, ma soprattutto della fotografia, che usa per farsi immortalare durante amplessi con donne destinate a morte certa (Brona), dalle quali, in una sorta di ribaltamento dell'amplesso vampiresco, si fa sputare addosso sangue infetto.

Dall'ucronia di Moore all'anacronismo di Logan si compie il percorso definitivo che conduce il mito letterario (e mediale) a cristallizzarsi in icona culturale, superando (o meglio: annullando) i confini e i contorni temporali e sociali, stratificando le contaminazioni e le ibridazioni, e dissolvendo progressivamente i caratteri identitari del testo originario.

Le icone di The League of Extraordinary Gentlemen e di Penny Dreadful sono oggetti, morbidi, permeabili, accoglienti, che consentono una proliferazione di sensi, interpretazioni, fruizioni. Sono malleabili e fluide: consentono di rileggere in termini critici l'ideologia del passato e di testarne il confronto con quella presente. Rievocando alcuni topoi compresenti alla letteratura alta (il romanzo vittoriano) e a quella popolare (il penny dreadful), come il femminile, la mostruosità, la doppiezza, la reazione all'alterità, la sessualità perversa, funzionano come vettori di interrogazione sociale.

In sintesi potremmo affermare che i due prodotti culturali in esame ben esemplificano una delle possibili modalità di generazione delle icone culturali

nel 1891, un libro a lui, in tutti i sensi, precedente: dopo qualche minuto la Creatura ribelle di Victor uccide Van Helsing, consentendogli di "rinascere", sei anni dopo, dalla penna di Stoker. 


\section{Ocula}

Vol 21, No 22 (April 2020) • DOI: 10.12977/ocula2020-15

Andrea Bellavita | Personaggio, mito, icona. A League of Extraordinary Gentlemen

e Penny Dreadful

contemporanee, che si organizza intorno ad una serie di processi di trasformazione e adattamento, a partire però da una "densità" dei testi di partenza, che garantisca una "tenuta" degli elementi semantici di base (personaggi, situazioni, mondi), ascrivibili a quelli che abbiamo definito miti a bassa intensità o meme culturali. Il primo processo è quello di de-temporalizzazione: nella forma dell'ucronia e dell'anacronismo (o, più in generale di tutte le modalità di aggiornamento che caratterizzano il period drama impuro) il tempo storico originario viene perturbato, e posto in coalescenza dialettica con quello presente, della fruizione da parte del destinatario mediale (lettore, spettatore). Il secondo, strettamente collegato, è quello di re-indiziarietà: il potenziale indiziario che i testi in esame sviluppavano nei confronti dell'epoca di apparizione (qui: il sistema della produzione letteraria, alta e bassa, ottocentesco) viene riletto in funzione del sistema socio-culturale contemporaneo. Spesso, ed è il terzo movimento, attraverso logiche di ribaltamento dei sistemi ideologici di partenza, non solo nella direzione di un avvicinamento al presente, ma anche di uno svelamento dei sottotesti rimossi nell'originale. Questi processi trasformativi poi si accompagano (in perfetta coerenza con le strategie narrative postmoderne) con altre due pratiche essenziali: quella dell'ibridazione e quella del trattamento ludico.

Un'attività creativa affascinante e feconda che, per sua stessa natura (e nel collocarsi all'interno delle pratiche editoriali e produttive della serialità, editoriale o audiovisiva), è in continua evoluzione: per una linea narrativa che sembra destinata alla chiusura definitiva (ma che trova declinazioni sempre nuove nell'opera di Moore), un'altra si riapre, con nuovi personaggi, nuovi tempi e nuove ambientazioni: Penny Dreadful: City of Angels, ambientata nella Los Angeles del 1938 è, attualmente, in produzione.

\section{Bibliografia}

Artt, Sarah

2018 "'An otherness that cannot be sublimated'. Shades of Frankenstein in Penny Dradful and Black Mirror", Science Fiction Film and Television, 11,2, 2018, pp. $257-75$.

Baptista do Lago, Izabela

2015 “A estética vitoriana transposta para a tela da televisão. Uma análise intermidiática do seriado Penny Dreadful", Revista Rascunhos Culturais, 6, 12, 2015, pp. 291-302.

Bellavita, Andrea

2019 "Ucronia meta-funzionale: Penny Dreadful e Once Upon a Time. L'ipertestualità come carattere dominante della fiction seriale contemporanea", in Brignoli L. (a cura di), Interartes. Diegesi migranti, Sesto San Giovanni, Mimesis, pp. 245-261.

Braid, Barbara

2017 "The Frankenstein Meme: Penny Dreadful and The Frankenstein Chronicles as Adaptation”, Open Cultural Studies, 1, 2017, pp. 232-243. 


\section{Ocula}

Vol 21, No 22 (April 2020) • DOI: 10.12977/ocula2020-15

Andrea Bellavita | Personaggio, mito, icona. A League of Extraordinary Gentlemen

e Penny Dreadful

Dawkins, Richard

1976 The selfish gene, London, Oxford University Press (tr. it. di D. Conti, T. Imbastaro, Il gene egoista, Bologna, Zanichelli, 1979).

Domsch, Sebastian

2012 "Monsters against Empire: The Poetics and Politics of Neo-Victorian Metafiction", in M.L. Kohlke, C. Gubleten (a cura di), The League of Extraordinary Gentlemen. Neo-Victorian Gothic: Horror, Violence and Degeneration in the Re-Imagined Nineteenth Century, Amsterdam, Rodopi, pp. 97-121.

Gagliardi, Lucas

2016 "El espejo de Pandora: identidad y monstruosidad en Penny Dreadful", Brumal. Research Journal on the Fantastic, IV, 1, 2016, pp. 35-56.

García Sahagún, Marta; Arquero Blanco, Isabel

2019 "La tematología aplicada al análisis de la serie Penny Dreadful", Comunicación y Sociedad, 2019, pp. 1-26, e666o. DOI: <https://doi.org/10.32870/cys. v2019io.6660>.

Jones, Jason B.

2010 "Betrayed by Time: Steampunk \& the Neo-Victorian in Alan Moore's Lost Girls and The League of Extraordinary Gentlemen", Neo-Victorian Studies 3.1, pp. 99-126.

Halsaal, Alison

2015 “'A Parade of Curiosities': Alan Moore’s The League of Extraordinary Gentlemen and Lost Girls as Neo-Victorian Pastiche", The Journal of Popular Culture, 48, 2, 2015, pp. 253-254.

Hutcheon, Linda

2006 A Theory of Adaptation, New York-London, Routledge (tr. it. di G. V. Distefano, Teoria degli adattamenti. I percorsi delle storie fra letteratura, cinema e nuovi media, Roma, Armando, 2011).

Lee, Alison; King, Frederick D.

2015 "From Text, to Myth, to Meme: Penny Dreadful and Adaptation", Cahiers victoriens et édouardiens, 82, 2015, <https://doi.org/10.4000/cve.2343>.

Mitchell, Rebecca N.

2017 "Before and After. Punch, Steampunk, and Victorian Graphic Narrativity", in Jones, A.M. e Mitchell, R.N. (eds.), Drawing on the Victorians. The Palimpsest of Victorian and Neo-Victorian Graphic Texts, Athens, Ohio University, pp. 237-268.

Mittel, Jason

2015 Complex Tv. The Poetics of Contemporary Television Storytelling, New York, New York University Press (tr. it. di M. Maraschi, Complex TV. Teoria e tecnica dello storytelling delle serie tv, Roma, Minimum Fax, 2017).

Nevins, Jess

2003 Heroes \& Monsters: The Unofficial Companion to The League of Extraordinary Gentlemen, Austin, MonkeyBrain. 


\section{Ocula ${ }^{22}$}

Vol 21, No 22 (April 2020) • DOI: 10.12977/ocula2020-15

Andrea Bellavita | Personaggio, mito, icona. A League of Extraordinary Gentlemen

e Penny Dreadful

Nevins, Jess

2004 A Blazing World: The Unofficial Companion to The League of Extraordinary Gentlemen, Volume Two, Austin, MonkeyBrain.

Ortoleva, Peppino

2019 Miti a bassa intensità. Racconti, media, vita quotidiana, Torino, Einaudi.

Pescatore, Guglielmo (a cura di)

2018 Ecosistemi narrativi. Dal fumetto alle serie tv, Roma, Carocci.

Rocha, Lauren

2016 "Angel in the House, Devil in the City: Explorations of Gender in Dracula and Penny Dreadful", Critical Survey and Berghahn Books, 28, 1, 2016, pp. 3039.

Springhall, John

1994 “'Diseeminating impure literature': the 'penny dreadful' publishing business since 1860”, Economic History Review, XLVIII, 3, 1994, pp. 567-584.

Sulmicki, Maciej

2011 "The Author as Antiquarian: Selling Victorian Culture to Readers of NeoVictorian Novels and Steampunk Comics", Otherness: Essays and Studies, 2,1, 2011, <http://www.otherness.dk/journal/vol-2>.

Toss, Jeff

2015 "From Penny Dreadful to Graphic Novel: Alan Moore and Kevin O’Neill's Genealogy of Comics in The League of Extraordinary Gentlemen”, Belphégor. Littérature Populaire et culture médiatique, 13, 1, 2015, <https://doi. org/10.4000/belphegor.624>.

Truman, Emily

2017 "Rethinking the Cultural Icon: Its Use and Funciton in Popular Culture", Canadian Journal of Communication, 42, 2017, pp. 829-849.

Wegner, Philipp E.

2010 “Alan Moore, 'Secondary Literacy,' and the Modernism of the Graphic Novel”, ImageText, 5, 3, 2010, <http://imagetext.english.ufl.edu/archives/v5_3/ wegner>.

\section{Filmografia}

The 39 Steps, 1935 (Il club dei 39), Alfred Hitchcock. Alien: Covenant, 2017 (Alien - Covenant), Ridley Scott. The Aviator, 2004 (The Aviator), Martin Scorsese.

Dangerous Liaisons, 1988 (Le relazioni pericolose), Stephen Frears.

Gladiator, 2000 (Il gladiatore), Ridley Scott.

Hugo, 2011 (Hugo Cabret), Martin Scorsese.

The Last Samurai, 2003 (L'ultimo samurai), Edward Zwick.

The League of Extraordinary Gentlemen, 2003 (La leggenda degli uomini straordinari), Stephen Norrington.

Penny Dreadful, 2014-2016 (Penny Dreadful), John Logan, Showtime.

Pretty Woman, 1990 (Pretty Woman), Garry Marshall.

Spectre, 2015 (Spectre), Sam Mendes. 


\section{Ocula ${ }^{22}$}

Vol 21, No 22 (April 2020) • DOI: 10.12977/ocula2020-15

Andrea Bellavita | Personaggio, mito, icona. A League of Extraordinary Gentlemen

e Penny Dreadful

Skyfall, 2012 (Skyfall), Sam Mendes.

Star Trek: Nemesis, 2002 (Star Trek - La nemesi), Stuart Baird.

Sweeney Todd: The Demon Barber of Fleet Street, 2007 (Sweeney Todd - Il diabolico barbiere di Fleet Street), Tim Burton.

\section{Opera letterarie (edizione consultata)}

Conan Doyle, Arthur

1893 The Final Problem, London, George Newnes (tr. it. di N. Rosati Bizzotto, L'ultima avventura, Roma, Newton Compton Editori, 2015).

Dickens, Charles

1870 The Mystery of Edwin Drood, London, Chapman \& Hall (tr. it. Il mistero di Edwin Drood, di P.F. Paolini, Milano, Rusconi, 1984).

Farmer, Philip José

1972 Tarzan Alive: A Definitive Biography of Lord Greystoke, New York, Doubleday.

Farmer, Philip José

1972 Doc Savage: His Apocalyptic Life, New York, Doubleday (tr. it. R. Valla, di. Milano, Doc Savage: una biografia apocalittica, Mondadori, 1992).

Farmer, Philip José

1973 The Other Log of Phileas Fogg, New York, Doubleday (tr. it. di R. Valla, Il diario segreto di Phileas Fogg, Milano, Arnoldo Mondadori Editore, 1990).

Fruttero, Carlo; Lucentini, Franco

1989 La verità sul caso D., Milano, Einaudi.

Rider Haggard, Henry

1885 King Salomon's Mines, London, Cassel and Company (tr. it. di G. Sesostra, Le miniere di Re Salomone, Milano, Universale Sonzogno, 1974).

Shelley, Mary

1818 Frankenstein; or, The Modern Prometheus, London, Lackington, Hughes, Harding, Mavor \& Jones (tr. it. di C. Zanolli, L. Caretti, Frankenstein o il moderno Prometeo, Milano, Arnoldo Mondadori Editore, 1986).

Somerset Maugham, William

1908 The Magician, London, Heinemann (tr. it. di O. Viani, Il mago, Milano, Arnaldo Mondadori Editore, 1968).

Stevenson, Robert Louis

1886 Strange Case of Dr Jekyll and Mr Hyde, London, Longmans Green \& Co (tr. it. di C. Fruttero, F, Lucentini, Lo strano caso del Dr. Jekyll e del Sig. Hyde, Milano, Einaudi, 1983).

Stoker, Bran

1897 Dracula, Archibald Constable and Company (tr. it. di A. Pellegrini, Dracula, Milano, Longanesi, 1966).

Verne, Jules

1869-70 Vingt Mille Lieues sous les mers, Pierre-Jules Hetzel (tr. it. di M.G. Schiuzzi, Ventimila leghe sotto i mari, Milano, Mursia, 1982). 


\section{Ocula ${ }^{22}$}

Vol 21, No 22 (April 2020) • DOI: 10.12977/ocula2020-15

Andrea Bellavita | Personaggio, mito, icona. A League of Extraordinary Gentlemen

e Penny Dreadful

Wells, Herbert George

1895 The Time Machine, London, Heinemann (tr. it. di R. De Michele, La macchina del tempo, Milano, Rizzoli, 1975).

Wells, Herbert George

1896 The Island of Doctor Moureau, London, Heinemann (tr. it. di M. A. Puddu, L’isola del Dottor Moureau, Milano, Mursia, 1978).

Wells, Herbert George

1897 The Invisible Man, London, C. Arthur Pearson (tr. it. di G. Gatti, L'uomo invisibile, T. Ribero, Milano, Bompiani, 1988).

Wells, Herbert George

1898 The War of the Worlds, London, William Heinemann (tr. it. di A. Motti, La guerra dei mondi, Milano, Mursia, 1965).

Wilde, Oscar

1891 The Picture of Dorian Gray, London, Ward Lock \& Company (tr. it. di R. Calzini, Il ritratto di Dorian Gray, Milano, Arnoldo Mondadori Editore, 1982).

Wolf, Virginia

1928 Orlando: A Biography, Richmond, Hogart Press (tr. it. di A. Scalero, Orlando, Milano, Garzanti, 1978).

Opere a fumetti (edizioni raccolte in volume)

Moore, Alan; Lloyd, Kevin David

1990 V for Vendetta, New York, Warner Books. (Ed. it. V for Vendetta, Novara, RW Lion, 2015); uscita originale periodica: 1982-1989, in Warrior, London, Quality Communications.

Moore, Alan; Campbell, Eddie

1999 From Hell, Marietta, GA, Top Shelf (Ed. it. From Hell, Roma, Magic Press, 2008); uscita originale periodica: 1989-1996, in Taboo, Princeton, WI, Kitchen Sink Press.

Moore, Alan; O’Neill, Kevin

2000 The League of Extraordinary Gentlemen Volume I, La Jolla, CA, Wildstorm - America's Best Comics. (Ed. it. La Lega degli Straordinari Gentlemen, Volume 1, Milano, Bao Publishing, 2019, nuova edizione); uscita originale periodica: 1999-2000, La Jolla, CA, Wildstorm - America's Best Comics.

Moore, Alan; O’Neill, Kevin

2003 The League of Extraordinary Gentlemen Volume II, La Jolla, CA, Wildstorm - America's Best Comics. (Ed. it. La Lega degli Straordinari Gentlemen, Volume 2, Milano, Bao Publishing, 2019, nuova edizione); uscita originale periodica: 2002-2003, La Jolla, CA, Wildstorm - America's Best Comics.

Moore, Alan; Burrows, Jacen

2004 Alan Moore's The Courtyard, Rantoul, IL, Avatar Press. (Ed. it. Il cortile, Roma, Magic Press, 2004); uscita originale periodica: 2003, Rantoul, IL, Avatar Press. 


\section{Ocula}

Vol 21, No 22 (April 2020) • DOI: 10.12977/ocula2020-15

Andrea Bellavita | Personaggio, mito, icona. A League of Extraordinary Gentlemen

e Penny Dreadful

Moore, Alan; Gebbie, Melinda

2006 Lost Girls, Marietta, GA, Top Shelf. (Ed. it. Lost Girls - Ragazze perdute, Vol 1-3, Roma, Magic Press, 2008); uscita originale periodica: 1991-1992, in Taboo, Princeton, WI, Kitchen Sink Press.

Moore, Alan; O’Neill, Kevin

2007 The League of Extraordinary Gentlemen: Black Dossier, La Jolla, CA, Wildstorm - America's Best Comics. (Ed. it., La Lega degli Straordinari Gentlemen: Black Dossier, Milano, Bao Publishing, 2013).

Moore, Alan; O'Neill, Kevin

2009 The League of Extraordinary Gentlemen: Century: 1910, Marietta, GA, Top Shelf. (Ed. it., in La Lega degli Straordinari Gentlemen, Volume 3 - Century, Milano, Bao Publishing, 2019, nuova edizione).

Moore, Alan; Burrows, Jacen

2011 Neonomicon, Rantoul, IL, Avatar Press. (Ed. it. Neonomicom, Milano, Bao Publishing, 2011); uscita originale periodica: 2010-2011, Rantoul, IL, Avatar Press.

Moore, Alan; O’Neill, Kevin.

2011 The League of Extraordinary Gentlemen: Century: 1969, Marietta, GA, Top Shelf. (Ed. it., in La Lega degli Straordinari Gentlemen, Volume 3 - Century, Milano, Bao Publishing, 2019, nuova edizione).

Moore, Alan; O’Neill, Kevin

2012 The League of Extraordinary Gentlemen: Century: 2009, Marietta, GA, Top Shelf. (Ed. it., in La Lega degli Straordinari Gentlemen, Volume 3 - Century, Milano, Bao Publishing, 2019, nuova edizione).

Moore, Alan; McLaren, Malcolm; Johnston, Antony

2012 Fashion Beast, Rantoul, IL, Avatar Press. (Ed. it. Fashion Beasts, Modena, Panini Comics, 2013); solo uscita periodica.

Moore, Alan; O’Neill, Kevin

2013 Nemo: Heart of Ice, Marietta, GA, Top Shelf. (Ed. it., in La Lega degli Straordinari Gentlemen, Nemo, Milano, Bao Publishing, 2020).

Moore, Alan; O’Neill, Kevin

2014 Nemo: Roses of Berlin, Marietta, GA, Top Shelf. (Ed. it., in La Lega degli Straordinari Gentlemen, Nemo, Milano, Bao Publishing, 2020).

Moore, Alan; O’Neill, Kevin

2015 Nemo: River of Ghosts, Marietta, GA, Top Shelf. (Ed. it., in La Lega degli Straordinari Gentlemen, Nemo, Milano, Bao Publishing, 2020).

Moore, Alan; Burrows, Jacen

2016 Providence Act 1, Rantoul, IL, Avatar Press. (Ed. it. Providence, Modena, Panini Comics, 2016); uscita originale periodica: 2015, Rantoul, IL, Avatar Press

Moore, Alan; Burrows, Jacen

2017 Providence Act 2, Rantoul, IL, Avatar Press. (Ed. it. Providence, Modena, Panini Comics, 2017); uscita originale periodica: 2016, Rantoul, IL, Avatar Press. 


\section{Dcula ${ }^{22}$}

Vol 21, No 22 (April 2020) • DOI: 10.12977/ocula2020-15

Andrea Bellavita | Personaggio, mito, icona. A League of Extraordinary Gentlemen

e Penny Dreadful

2017 Providence Act 3, Rantoul, IL, Avatar Press. (Ed. it. Providence, Modena, Panini Comics, 2018); uscita originale periodica: 2017, Rantoul, IL, Avatar Press.

Moore, Alan; O'Neill, Kevin

2019 The League of Extraordinary Gentlemen Volume IV: Tempest, Marietta, GA, Top Shelf.

Andrea Bellavita è Professore associato di Storytelling e forme del racconto mediale e Factual entertainment e scrittura della realtà e Vicedirettore del corso di laurea di Storia e storie del mondo contemporaneo presso l'Università dell'Insubria di Varese. Redattore di "Segnocinema", collabora a "Film TV", "Cineforum", "8 e 1/2". È autore di vari libri e saggi sul rapporto tra cinema e psicoanalisi. La sua attività di ricerca oggi è focalizzata sulla fiction seriale e sulle strategie di adattamento transmediali. 